\title{
BMJ Open Adequacy and timeliness of antenatal care visits among Ethiopian women: a community-based panel study
}

\author{
Kasiye Shiferaw (D) , ${ }^{1}$ Bezatu Mengistie, ${ }^{2}$ Tesfaye Gobena, ${ }^{3}$ Merga Dheresa, ${ }^{1,4}$ \\ Assefa Seme $^{5}$
}

To cite: Shiferaw K,

Mengistie B, Gobena T, et al. Adequacy and timeliness of antenatal care visits among Ethiopian women: a communitybased panel study. BMJ Open 2021;11:e053357. doi:10.1136/ bmjopen-2021-053357

- Prepublication history for this paper is available online. To view these files, please visit the journal online (http://dx.doi. org/10.1136/bmjopen-2021053357).

Received 11 May 2021 Accepted 30 November 2021

Check for updates

(c) Author(s) (or their employer(s)) 2021. Re-use permitted under CC BY-NC. No commercial re-use. See rights and permissions. Published by BMJ.

${ }^{1}$ School of Nursing and Midwifery, Haramaya University College of Health and Medical

Sciences, Harar, Ethiopia

${ }^{2}$ School of Public Health, St

Paul's Hospital Millennium

Medical College, Addis Ababa,

Ethiopia

${ }^{3}$ Environmental Health Science, Haramaya University College of Health and Medical Sciences,

Harar, Ethiopia

${ }^{4}$ Public Health, Addis Continental Institute of Public Health, Addis Ababa, Ethiopia

${ }^{5}$ School of Public Health, Addis Ababa University, Addis Ababa, Ethiopia

Correspondence to

Dr Kasiye Shiferaw;

sifkas.gem2@gmail.com

\section{ABSTRACT}

Objectives The purposes of the study are; (A) to identify whether Ethiopian women's antenatal care (ANC) visits are adequate and timely and (B) to explore factors that determine these.

Design Panel study design.

Setting Ethiopia.

Participants A total of 2855 women nested within 217 enumeration areas.

Primary outcome measures Adequacy and timeliness of ANC visits.

Results Of all the 2855 respondents, $65 \%$ had made an ANC visit once, while $26.8 \%$ initiated ANC visits in a timely way and $43.3 \%$ attended adequate ANC visits. Rural residence $($ adjusted $\mathrm{OR}(\mathrm{AOR})=0.55,95 \% \mathrm{Cl}: 0.36$ to 0.84 ) attending higher level of education $(\mathrm{AOR}=2.64,95 \% \mathrm{Cl}$ : 1.47 to 4.77 ), being multipara (AOR $=0.53,95 \% \mathrm{Cl}: 0.32$ to 0.89 ) and encouragement by partners to attend clinic for ANC (AOR=1.98, 95\% Cl: 1.14 to 3.44) were significantly associated with timeliness of ANC visit. Similarly, residing in rural areas ( $\mathrm{AOR}=0.20,95 \% \mathrm{Cl}: 0.12$ to 0.35 ), attending higher level of education ( $\mathrm{AOR}=2.96,95 \% \mathrm{Cl}: 1.38$ to 6.15), encouragement by partners to attend clinic for ANC (AOR=2.11, 95\% Cl: 1.31 to 3.40) and timeliness of ANC visit ( $A O R=4.59,95 \% \mathrm{Cl}: 2.93$ to 7.21 ) were significantly associated with adequacy of ANC visits.

Conclusions A quarter of the pregnant women started ANC visits during the first trimester and nearly half attended adequate ANC visits with wider disparities across regions of their origin and their background characteristics. Concerted efforts on tailored interventions for rural residents, female education and partner involvement are recommended for early and adequate ANC visit(s).

\section{INTRODUCTION}

Although several countries pledged to reduce the maternal mortality ratio to less than 70 per 100000 live births by 2030, ${ }^{1}$ and set a plan to reduce newborn deaths to 10 and fewer per 1000 live births by $2035,{ }^{2}$ the current progress is not promising to achieve this goal. Ensuring access and quality maternal healthcare for all women is needed to improve maternal and newborn health. ${ }^{3}$ Appropriate antenatal care (ANC) practices that are timely have the lifesaving potential for the child and the mother ${ }^{4}$

\section{Strengths and limitations of this study}

A community-based panel study design was implemented.

- The utilisation of nationally representative data and sample weighting make the findings generalisable to women of reproductive age in Ethiopia.

- The use of a multilevel logistic regression model which considered the hierarchical nature of the data that included women nested within enumeration areas likely makes the results more conservative inference for the aggregate effect.

- The lost to follow-up, together with recall error as well as the tendency to give desirable information by women, could result in bias in the findings.

and prepare women for birth. ${ }^{5}$ The perinatal outcome could be improved by early identification (ID) and management of women's complications ${ }^{6}$ since these happen mostly during the antepartum period. ${ }^{7}$

Early ANC check-up facilitates women's meeting of the WHO recommendations, ${ }^{8}$ and is the major requirement of WHO's positive pregnancy experience. ${ }^{89}$ Specific to lowincome countries, WHO recommended early ANC initiation and a minimum of four ANC visits for effective ANC services. ${ }^{10}$ This recommendation has been implemented in Ethiopia, ${ }^{11}$ although a recommended minimum number of ANC contacts was updated to eight by WHO to improve women's care experience and reduce perinatal deaths. ${ }^{12}$ Accordingly, the first two ANC visits take place in the first two trimesters, and the last two visits should take place in the third trimester. ${ }^{13}$

Globally, although ANC contact remains high at $86 \%,{ }^{14}$ a significantly lower percentage of pregnant women initiate ANC in the first trimester. ${ }^{15}$ The estimated coverage of early ANC visits was $59 \%$ worldwide and $24 \%$ in low-income and middle-income countries (LMICs) in 2013. ${ }^{15}$ Studies revealed that pregnant women initiate their first ANC visit 
late, that is, during the second or third trimester of gestation. ${ }^{16-18}$ Similarly, $64 \%$ of pregnant women in Ethiopia initiate ANC late, ${ }^{17}$ and this is a major public health issue. ${ }^{20} \mathrm{~A}$ significant proportion of women receive at least four ANC visits across LMICs, ${ }^{21}$ which comprises the coverage of at least 4 ANC, ranging from $25 \%$ to $76 \%$, in sub-Saharan Africa, ${ }^{22}$ while only four in ten pregnant women have four ANC visits in Ethiopia. ${ }^{23}$ This means the recommended ANC utilisation remains suboptimal and performs poorly in Ethiopia. ${ }^{24}$ The timing, sufficiency, and content of ANC should be examined for ANC to be deemed effective. ${ }^{25}$ The content of care for pregnant women is independently affected by the timing of the first ANC check-up.8

Studies across LMICs identified that maternal educational status, ${ }^{9} 23242627$ age, ${ }^{28}$ marital status, ${ }^{29}$ economic levels, ${ }^{28}$ place of residence, ${ }^{2326}{ }^{30}$ parity, ${ }^{31}$ pregnancy intention, ${ }^{31}$ mass-media exposure,${ }^{30}$ distance from health facilities ${ }^{32}$ and regions ${ }^{30}$ are associated with timeliness of ANC visits. Similarly, maternal age, ${ }^{33} 34$ educational level, ${ }^{33}$ wealth quintile, ${ }^{33} 34$ place of residence, ${ }^{34}$ parity ${ }^{17}$ and frequency of watching television ${ }^{34}$ are associated with having four or more ANC visits. The exact mechanism through which these factors affect ANC visits, both in terms of timeliness and adequacy of ANC attendance, is unknown. ${ }^{17}$ For countries to adopt better intervention strategies and to understand where drop-out occurs or why women stop using maternal health services and who drops out is vital. Adequate ANC visits play an essential role within the continuum of care which mandates programmatic attention. $^{35}$

Previous studies did not consider how timely initiation of ANC affects the number of ANC. ${ }^{36}$ The majority of the previous studies in Ethiopia, regarding timeliness and adequacy of ANC visits, were facility-based crosssectional studies that rely on small sample sizes in a few regions. ${ }^{16} 1937$ Besides, the few studies conducted in Ethiopia used Demographic Health Survey (DHS) data, that is, relied on respondents' self-report of things that happened in the past 5 years. ${ }^{1724}{ }^{26}$ However,this study uses nationally representative panel data to determine the timeliness and adequacy of ANC visits and the associated factors. The study may come up with results that help policy-makers in the tailored execution of interventions that improve timely initiation and the number of ANC visits. Moreover, the present work also could contribute to the promotion of maternal and neonatal health in Ethiopia, at large.

\section{MATERIALS AND METHODS}

\section{Study design, area and period}

A panel study design was employed with pregnant and currently postpartum women (ie, women who followed their pregnancy to 6 weeks post partum). This study was conducted in five regions (ie, Tigray, Afar, Amhara, Oromia, and South Nation, Nationalities and People, SNNP) and one city administration (Addis Ababa) which collectively represent $90 \%$ of Ethiopia's population. ${ }^{38}$ Ethiopia is one of the countries in the horn of Africa with an estimated population of more than 106 million with 2.05 and 4.6 growth and fertility rate, respectively, as projected based on the 1994 census. ${ }^{39} 40$ The study period was from October 2019 to September 2020.

\section{Participants}

All women of reproductive age (15-49 years) were the source population. The study population included pregnant or currently postpartum women residing in the selected regions.

\section{Sampling population}

A multistage cluster design with urban-rural and major regions as strata was used to draw a probability sample of households and women of reproductive age. A probability proportional to size within the strata was used to draw all enumeration areas (EAs). ${ }^{38} \mathrm{EA}$ is a geographical area that has a varying number of households and is adopted from the Ethiopian Central Statistical Agency. EAs were drawn separately from rural and urban strata within Tigray, Amhara, Oromia and SNNP, while they were randomly selected with probability proportional to size within Afar without rural or urban stratification. All EAs were drawn from Addis Ababa without urban or rural stratification since it is exclusively urban.

A full census of the selected EAs was undertaken, listing names, sex and ages of all household members in the six regions in which cross-sectional and panel studies were implemented. The women of reproductive age within each household consented and were screened for eligibility in the panel study. This population served as the sampling frame for the panel study. Any eligible woman in the selected EAs was enrolled. Women who were regular members of the household, or those staying at their parents' home for pregnancy or postpartum period, currently pregnant or less than 8 weeks post partum with live birth, those within the age of 15-49 years, and living in panel regions, were eligible. Visitors were excluded from the study.

\section{Sample size determination}

Approximately $10 \%$ of the participant women of reproductive age were pregnant or currently postpartum (less than 6 weeks) based on previous rounds of PMA Ethiopia data. Furthermore, 98\% of women of 15-49 years of age per household and average EAs size of 175 were assumed. Both estimations were based on previous PMA Ethiopia rounds data. Finally, a sample size of 217 EAs in the regions was received as adequate for detecting a $5 \%$ difference between the group of interest for outcome. ${ }^{38}$

\section{Variables}

The outcome variables were categorised as timely initiation of first ANC visit (within 3 months of gestation=timely and beyond 3 months of gestation=late) and adequate ANC visits (having four plus ANC visits=adequate and less than four=inadequate). In addition, we 
assessed community-level factors, that is, place of residence (urban, rural), region (Tigray, Afar, Amhara, Oromia, SNNP, Addis Ababa), wealth quintile (lowest, lower, middle, higher, highest), community poverty level (low, high), and community illiteracy level (low, high). The individual-level factors considered in the analysis were: maternal age (15-19, 20-24, 25-29, 30-34, 35-39, 40-49 years), religion (Orthodox Christianity, Protestant, Muslim, others), educational level (never attended, primary, secondary, vocational and technical, higher), marital status (married, others), current pregnancy desired (then, later, not at all), marriage history (once, more than once), birth events (not given birth, given birth to $1-4$, given birth to $5+$ ), husband or partner has other wives or women (yes, no), ever been pregnant (yes, no) and encouragement by a partner to go to a clinic for ANC (yes, no). The proportion of women from the two lowest wealth quintiles was categorised as high if the proportion was $43 \%-100 \%$ and low if the proportion was $0 \%-42 \%$ to estimate community poverty level. Similarly, if the proportion of women who never attended school in the community was $67 \%-100 \%$, the community level illiteracy was high. Otherwise, it was considered as low. Previous studies also used this approach. ${ }^{41} 42$

\section{Data source}

The baseline survey for panel data on pregnant or currently postpartum women was conducted by Addis Ababa University School of Public Health in collaboration with Johns Hopkins Bloomberg School of Public Health with financial support by the Bill \& Melinda Gates Foundation through a grant to the Bill \& Melinda Gates Institute for Population and Reproductive Health. The survey project was designed to generate data on a variety of reproductive, maternal, and health indicators to inform the government and to fill the information gap, especially issues that were not measured by other large-scale surveys.

Female resident enumerators (REs) who were trained in collecting data using smartphone technologies mapped and listed every household within the EAs to create a sampling frame from which women were screened for eligibility. The REs explained the purpose of the survey and the cadence of interviews during screening. After census and female screening were completed, all eligible consented women of 15-49 years of age in the EAs were enrolled and provided an ID card that was filled with relevant information. A household questionnaire and female questionnaire interviews were conducted, resulting in baseline data collection across a range of gestational ages, from less than 1-month pregnant to 8 weeks post partum. The study participants were contacted twice during the panel study, that is, during pregnancy or at less than 5 weeks post partum for baseline data interview. Further, those who were pregnant or less than 5 weeks post partum during enrolment were contacted at 5-8 weeks post partum for a 6-week interview. To fill the 6-week interviews for the enrolled pregnant women, a list of the enrolled women was given to the health development army (HDA), that is, community volunteers who are not formally integrated into the health system but involved in seasonal activities for a limited time. The HDA called REs when pregnant women gave birth. Further, REs remained in contact with the HDA.

\section{Quality assurance}

The questionnaire was developed based on a DHS and literature review contextualising the country's reproductive, maternal and newborn health gaps. Open-source software for collecting and managing data (open data kit $(\mathrm{ODK})$ ) was used after programming the questionnaire. REs who had experience in DHS data collection and previous PMA Ethiopia panel study performed in SNNP during 2016 were recruited. Both supervisors and data collectors took 2 weeks of training on an in-depth review of the protocol, questionnaire contents, use of ODK, mobile data collection, and interview skills. Pretesting was conducted using a tool on the smartphone before data collection.

\section{Data processing and analysis}

STATA V.16.1 software was used for data analysis. To adjust for the study design used and non-response, the 'svy' command for survey data in STATA was used. We applied sample weights (female and household) to compensate for unequal probability selection of clusters and women to minimise selection and non-response bias. The sample weights in this study were the output of the inverse of EA and household selection probability and the inverse of household and female response rate. The basic characteristics of the participants were tabulated using frequencies and percentages. We checked the missing values, lost to follow-up and reasons, hence it is not a threat to validity. Eligible women were tracked during follow-up and were located by using mobile apps. The women were provided mobile card incentives and reminded to minimise loss to follow-up. The variance inflation factors (VIF) showed no collinearity of explanatory variables $(\mathrm{VIF}=1.32)$. The explanatory variables, which showed significant associations with timeliness and adequacy of ANC visits were entered in the multilevel logistic regression model. The process considered the hierarchical nature of the data since it included women nested within EAs. We fitted the null model (model without explanatory variable) to the final model (model with both the individual and community level variables) to identify community and individuallevel factors which were associated with timely initiation and adequate ANC visits using 'melogit' code of STATA. Likewise, Akaike's information criterion (AIC) was used to compare the models, and the final model was selected based on the smallest value of the information criterion.

Intraclass correlation coefficient (ICC), a proportional change in variance $(\mathrm{PCV})$ and median OR (MOR) ${ }^{43} 44$ were computed to measure random effect variations. The proportion of variance was explained by consecutive models, and the degree of variation of timely initiation of first ANC visit across clusters was described in terms of 
the OR scale. The variation between individuals within the same cluster was measured by ICC, and calculated by using: ICC $=\frac{\mathrm{VC}}{\mathrm{VC}+\frac{\pi 2}{3}}=\frac{\mathrm{VC}}{\mathrm{VC}+3.29}$ formula, where $\mathrm{V}_{\mathrm{C}}$ is the estimated variance in each model as estimated elsewhere ${ }^{44}$; that is, cluster level variance divided by total variance. We also measured total variation attributed to community and/or individual level factors at each model using PCV which was calculated as $P C V=\frac{\mathrm{VC}-\mathrm{VB}}{\mathrm{VC}}$, where $\mathrm{V}_{\mathrm{A}}$ variance of the initial model, and $\mathrm{V}_{\mathrm{B}=}$ variance of the model with more variables. ${ }^{44}$

The MOR between the individual of higher and lower propensity,forcomparing twoindividualsfrom twodifferent randomly chosen clusters, measured the unexplained cluster heterogeneity and computed using the formula: $M O R=\exp (\sqrt{(2 \times V C \times 0.6745))} \approx \exp (0.95 \sqrt{\mathrm{VC}})$

, where $\mathrm{V}_{\mathrm{C}}$ is the cluster level variance. ${ }^{44}{ }^{45}$ There is no variation between clusters if the MOR is $1{ }^{46}$ Adjusted OR (AOR) with a $95 \%$ CI was used to report the association, and a $\mathrm{p}<0.05$ was considered statistically significant.

\section{Patient and public involvement}

The public was not involved in the design or conducting, or reporting, or dissemination plans of the research.

\section{RESULTS}

\section{Characteristics of participants}

From 13192 participants who were interviewed during screening for eligibility, 2855 women were eligible and enrolled in the panel study. Of this, 2665 postpartum (less than 6 weeks) respondents were accessed during the second round of the interview and others were unreachable (with a $93.4 \%$ response rate). The mean age of the participants was $27.10( \pm 5.95)$ years. Among the respondents, $31.81 \%, 73.11 \%$ and $42.88 \%$ were aged $24-29$ years, resided in rural areas, and lived in the Oromia region, respectively. Furthermore, those who never attended school $(36.94 \%)$, were married $(95.19 \%)$ and were in the highest wealth quintile $(24.40 \%)$ accounted for the majority of the participants. The majority (66.34\%) of the women reported that the current pregnancy was desired, and they were multipara women $(61.50 \%)$ (table 1$)$. For further refer to previous publication. ${ }^{47}$

\section{Adequacy and timeliness of ANC visit}

In our study, $1855(64.97 \%)$ women reported that they had at least one ANC visit from skilled attendants during their current pregnancy. Accordingly, 503 (27\%; 95\% CI: $25 \%$ to $29 \%$ ) women initiated ANC visits during the first trimester of gestation. Among those who initiated late, $1200(65 \%)$ of the pregnant women initiated ANC in the second trimester of their pregnancy; nearly one in ten women $(8.30 \%)$ initiated the first ANC visit in their third trimester of pregnancy in Ethiopia.

The prevalence of adequate ANC visits among pregnant or currently postpartum women was 723 (43\%; $95 \%$ CI: $41 \%$ to $46 \%$ ) in Ethiopia (figure 1).
Table 1 Frequency distribution of women's characteristics in Ethiopia, 2020 ( $n=1855)$

\begin{tabular}{|c|c|c|}
\hline Variable & $\begin{array}{l}\text { Un-weighted } \\
\text { frequency (\%) }\end{array}$ & $\begin{array}{l}\text { Weighted } \\
\text { frequency (\%) }\end{array}$ \\
\hline \multicolumn{3}{|l|}{ Age } \\
\hline $15-19$ & $165(8.89)$ & 203 (10.99) \\
\hline $20-24$ & $473(25.61)$ & 450 (24.34) \\
\hline $25-29$ & $614(33.15)$ & $589(31.81)$ \\
\hline $30-34$ & $333(17.95)$ & $330(17.84)$ \\
\hline $35-39$ & $214(11.64)$ & $216(11.78)$ \\
\hline $40-49$ & $51(2.75)$ & $60(3.25)$ \\
\hline \multicolumn{3}{|l|}{ Community poverty level } \\
\hline Low & $1211(65.28)$ & $1144(61.75)$ \\
\hline High & $644(34.72)$ & 709 (38.25) \\
\hline \multicolumn{3}{|l|}{ Community illiteracy level } \\
\hline Low & $1460(78.71)$ & $1401(75.63)$ \\
\hline High & 395 (21.29) & $452(24.37)$ \\
\hline \multicolumn{3}{|l|}{ Religion } \\
\hline Orthodox & 978 (52.72) & $783(42.26)$ \\
\hline Muslim & $488(26.31)$ & $630(33.97)$ \\
\hline Protestant & $363(26.31)$ & $398(21.48)$ \\
\hline Others* & $26(1.40)$ & $43(2.30)$ \\
\hline \multicolumn{3}{|l|}{ Educational status } \\
\hline Never attended & $577(31.21)$ & $682(36.94)$ \\
\hline Primary & $664(35.85)$ & 734 (39.67) \\
\hline Secondary & $345(18.71)$ & $256(13.91)$ \\
\hline Technical and vocation & $97(5.23)$ & $75(4.06)$ \\
\hline Higher & $167(9.00)$ & $100(5.42)$ \\
\hline \multicolumn{3}{|l|}{ Current pregnancy desired } \\
\hline Then & $1055(70.81)$ & $984(66.34)$ \\
\hline Later & 344 (23.09) & $397(26.70)$ \\
\hline Not at all & $91(6.11)$ & 103 (6.96) \\
\hline
\end{tabular}

${ }^{*}$ Catholic or traditional or wakefata.

Factors associated with adequacy and timeliness of ANC visit Multivariable analysis revealed that the women's residence, region of dwelling, religion, level of education, birth events and partner involvement had significant associations with the timeliness of their ANC visits. Community, individual and both community and individual-level factors were fitted by using models I, II and III, respectively. Finally, a model with the least AIC value has been selected.

The odds of timely initiation of ANC visits were lower by $45 \%$ among rural residents compared with the urban (AOR $=0.55,95 \%$ CI: 0.36 to 0.84 ). The SNNP women had $67 \%$ lower odds of attending ANC in the first trimester of pregnancy compared with Tigray women $(\mathrm{AOR}=0.33$, 95\% CI: 0.16 to 0.67$)$, whereas the odds of initiation of first ANC visit in the first trimester among Addis Ababa women was twice that of Tigray region $(\mathrm{AOR}=2.07,95 \%$ 


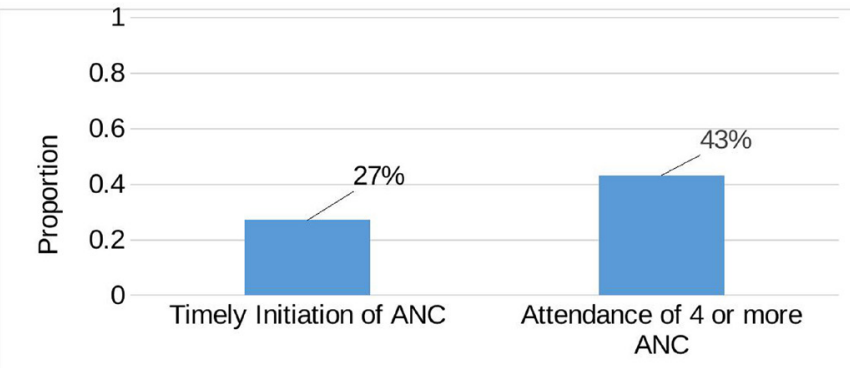

ANC visit

Figure 1 Timeliness and adequacy of ANC visits among women in Ethiopia $2020(n=1855)$. ANC, antenatal care.

CI: 1.02 to 4.21$)$. The odds of timeliness of ANC visit was $50 \%$ lower among Muslim women compared with their Orthodox counterparts $(\mathrm{AOR}=0.50,95 \%$ CI: 0.31 to $0.80)$.

The educational status of the women played a key role in the initiation of $\mathrm{ANC}$ visit in the first trimester of pregnancy. The odds of initiating ANC visit in the first trimester of pregnancy among women who attended vocational and technical education were twice that of those who never attended formal education (AOR $=2.21,95 \%$ CI: 1.11 to 4.45$)$. Similarly, women who attended higher education had increased odds of initiating ANC visit in the first trimester of pregnancy compared with women who never attended formal education $(\mathrm{AOR}=2.64,95 \%$ CI: 1.47 to 4.77$)$.

The odds of initiating ANC during the first trimester of pregnancy among multipara women were $47 \%$ lower compared with primipara women (AOR=0.53, 95\% CI: 0.32 to 0.89 ). A woman who was encouraged by her partner to go to a clinic for an ANC visit had increased odds of initiating ANC in the first trimester of pregnancy compared with her counterpart who did not get such encouragement $(\mathrm{AOR}=1.98,95 \% \mathrm{CI}: 1.14$ to 3.44$)$ (table 2).

Similarly, multivariable analysis showed that the women's place of residence, region, religion, educational status, encouragement by their partners to go to a clinic for ANC, and timely initiation of ANC visits had a significant association with adequate ANC visits

Women who lived in a rural part of Ethiopia had $80 \%$ lower odds of four or more ANC visits compared with those who lived in urban (AOR=0.20, 95\% CI: 0.12 to $0.35)$. Likewise, the geographical location of the women also played important role in the utilisation of four or more ANC visits. A woman in Afar (AOR=0.03, 95\% CI: 0.01 to 0.09 ) or SNNP (AOR=0.31, $95 \% \mathrm{CI}$ : 0.13 to 0.79 ) had $97 \%$ and $69 \%$ lower odds of using four or more ANC. visits compared with those who were in Tigray region, whereas Addis Ababa women had higher odds of four or more ANC visits utilisation $(\mathrm{AOR}=1.82,95 \% \mathrm{CI}: 1.30$ to 5.03).

Further, as the educational level of the women increased the odds of using four or more ANC increased. Hence, the odd of four or more ANC among those who attended secondary education was twice that of those who never attended formal education (AOR $=2.81,95 \%$ CI: 1.56 to 5.06). Those who attended technical and vocational level education also had increased odds of four or more ANC visits (AOR=5.07, 95\% CI: 1.81 to 14.43$)$. Similarly, women who attended higher education had increased odds of four or more ANC visits compared with those who never attended formal education $(\mathrm{AOR}=2.96,95 \%$ CI: 1.38 to 6.15 ).

The odds of four or more ANC visits among women who were encouraged by their partners to go to ANC clinic were twice that of their counterparts who were not encouraged to go to the clinic $(\mathrm{AOR}=2.11,95 \%$ CI: 1.31 to 3.40). Those who initiated ANC in the first trimester of pregnancy had increased odds of attending four or more ANC compared with their counterpart who did not initiate it (AOR=4.59, 95\% CI: 2.93 to 7.21) (table 3).

\section{Measures of variation and model-fit statistics}

In the null model, timely initiation of ANC across the clusters showed significant variation $(\tau=0.98, \mathrm{p}<0.05)$, in which $27 \%$ variation in the odds of timely initiation of ANC was explained by cluster-level factors $(\mathrm{ICC}=27 \%)$. After adjusting for community factors, it was found that the variation in the odds of timely initiation of ANC remained statistically significant across the community $(\tau=0.31, \mathrm{p}<0.05)$. With $39.8 \%$ variation in the odds of timely initiation, ANC was attributed to the communitylevel factors, and $11 \%$ of the variation among clusters was attributed to community-level factors after adjusting the model I for community-level factors.

Similarly, after adjusting to Model II for individuallevel factors, we observed that the variation in the odds of timely initiation of ANC was not statistically significant across the communities $(\tau=0.79, \mathrm{p}<0.05)$. With a $7.6 \%$ variation in the odds of timely initiation, the ANC was attributed to the individual-level factors, and $21 \%$ variation in the timely initiation of ANC visit was attributed to community-level factors. Model III (best-fit model), which was adjusted to both community and individuallevel factors showed statistically significant variation to the odds of timely initiation of ANC visits $(\tau=0.43$, $\mathrm{p}<0.05)$. About $12 \%$ of the variability among communities in timely initiation of ANC was due to communitylevel factors, and $35.1 \%$ of the variance in odds of timely initiation of ANC visits across communities was attributed to both individual and community-level factors.

On the other hand, there was a statistically significant variation of adequate ANC visits across the communities $(\tau=2.4, \mathrm{p}<0.05)$ in the null model, in which $44 \%$ variation in the odds of adequate ANC visits was attributed to community-level factors. The variation in the odds of adequate ANC visits was statistically significant across the communities $(\tau=0.79, \mathrm{p}<0.05)$. Also, the $42.4 \%$ variation in the odds of adequate ANC visits was attributed to the community-level factors. However, after adjusting the model for community-level factors in model I, it 
Table 2 Multilevel logistic regression analysis of timeliness of ANC visit by Ethiopian women and associated factors, 2020 $(n=1855)$

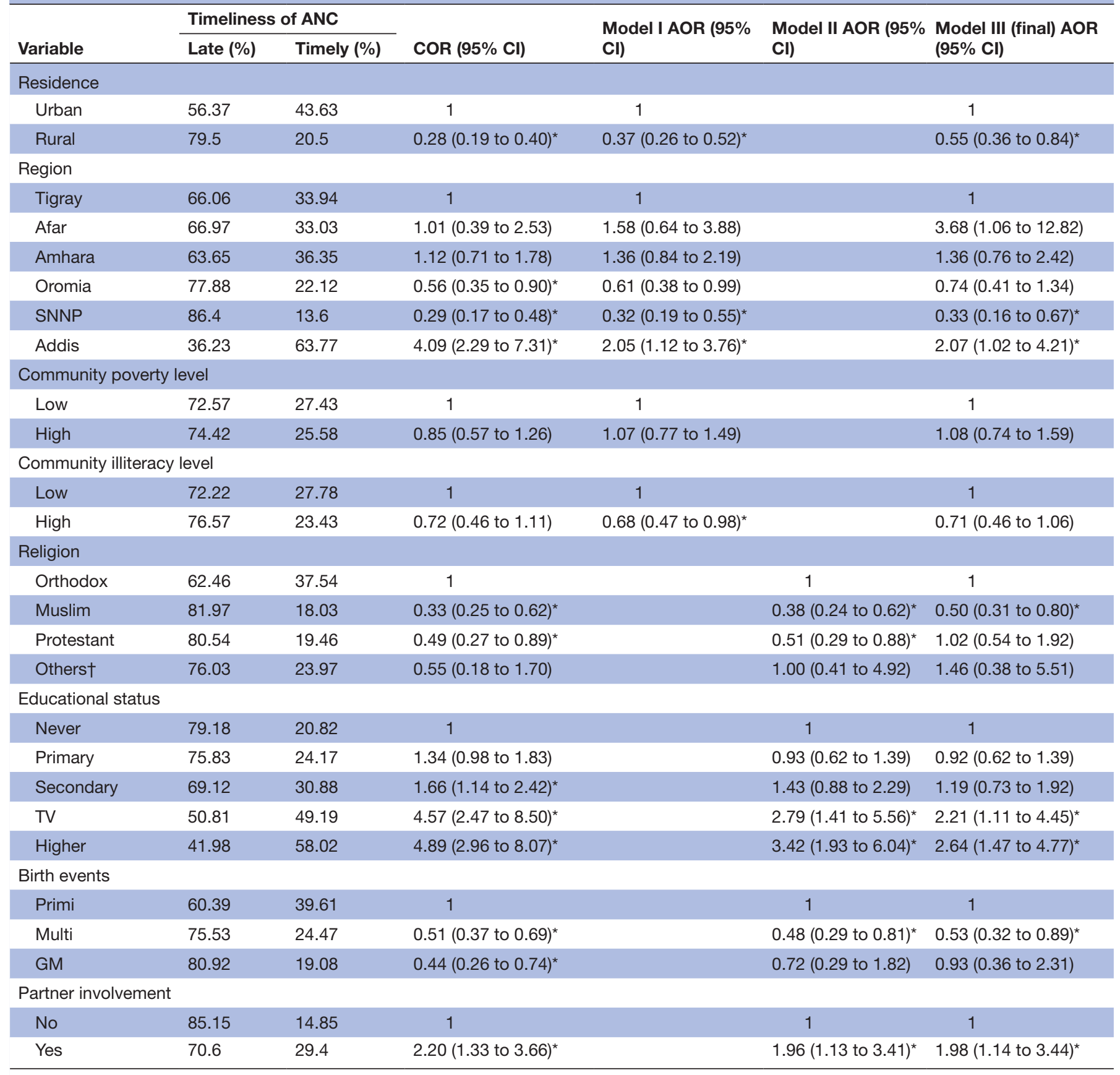

${ }^{*} \mathrm{P}<0.05$.

†Catholic or traditional or wakefata.

ANC, antenatal care; AOR, adjusted OR ; COR, crude odds ratio; GM, grand multipara; SNNP, South Nation, Nationalities and People; TV, television.

was found that $20 \%$ of the variation was attributed to community-level factors.

Moreover, the model adjustment was carried out for individual factors (model II), and the variation in odds of four or more ANC visits remained statistically significant $(\tau=1.6, \mathrm{p}<0.05)$ across the communities compared with variance in the null model. The community and individual-level factors indicated that $20 \%$ of the variability in the odds of adequate ANC visits and 32\% of the variation among the clusters were both attributed to community-level factors. Model III (best-fit model), which was adjusted for both community and individuallevel factors, showed statistically significant variation to the odds of adequate ANC visits $(\tau=0.90, \mathrm{p}<0.05)$. About $22 \%$ of the variability among communities in four or more ANC visits was due to community-level factors, and $35.1 \%$ of the variance in odds of adequate ANC visits across communities was attributed to both individual and community-level factors. 
Table 3 Multilevel mixed-effect analysis of factors associated with adequacy of ANC visits among Ethiopian women, 2020 $(n=1855)$

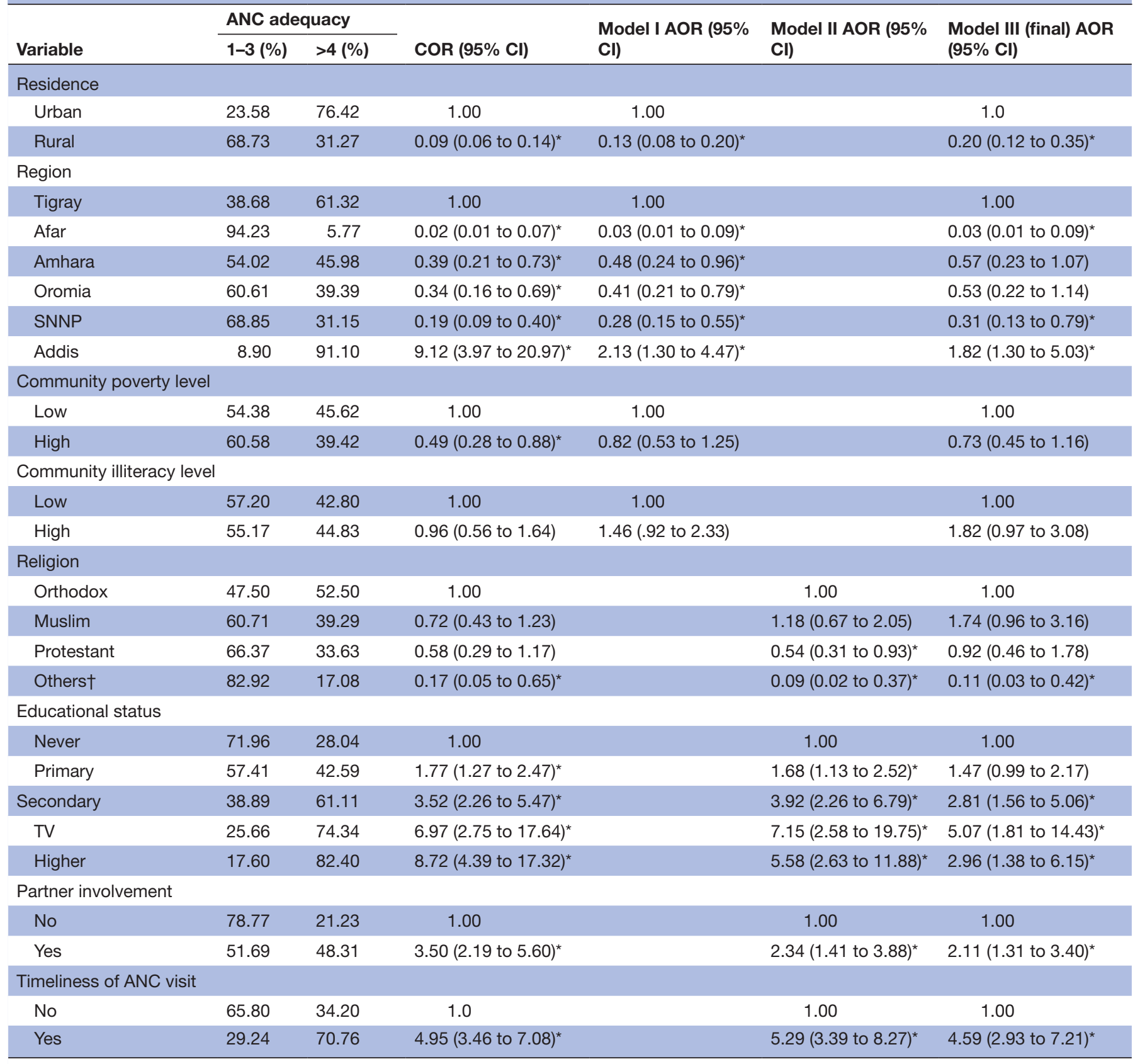

${ }^{*} \mathrm{P}<0.05$.

†Catholic or traditional or wakefata $\ddagger$

ANC, antenatal care; AOR, adjusted OR; COR, crude odds ratio; GM, grand multipara; SNNP, South Nation, Nationalities and People; TV, technical and vocational educational level; TV, technical and vocational educational level.

The null model showed the highest MOR value (2.6). What this means is that when an individual is randomly selected from a cluster with a higher timely initiation of first ANC visit and when the other was selected from a cluster at lower timely initiation of first ANC visit, individuals at the cluster with a higher timely initiation of first ANC visit had 2.6 times higher odds of timely initiation of ANC visit as compared with their counterparts. Similarly, the null model had the highest MOR value (3.3) which means that when an individual is randomly selected from a cluster with adequate $\mathrm{ANC}$ visits and the other from a cluster with inadequate $\mathrm{ANC}$ visits, those at the cluster with adequate ANC visits had 3.3 times higher odds of adequate ANC visits as compared with their counterparts (table 4).

\section{DISCUSSION}

Results of this study reveal that timely initiation and adequate ANC visits are low in Ethiopia. The analyses 
Table 4 Measures of variation and model-fit statistics in timeliness and adequacy of ANC visits among Ethiopian women, 2020

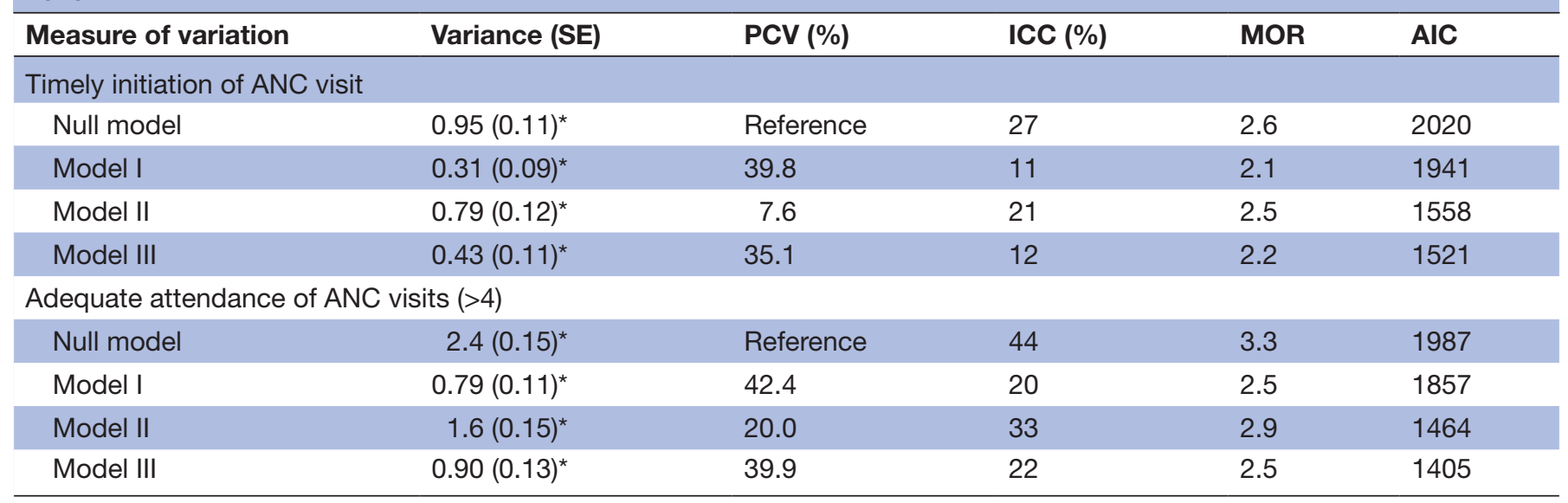

Model I-adjusted for community-level factors.

Model II-adjusted for individual-level factors.

Model III- a final model adjusted for both individual and community-level factors.

Here, a null model is an empty model, a baseline model without any explanatory variable.

${ }^{*} \mathrm{P}<0.05$.

AIC, Akaike's information criterion; ANC, antenatal care; ICC, intraclass correlation coefficient; MOR, median OR; PCV, proportional change in variance.

show that rural residence, educational status and encouragement by partners to go to a clinic for ANC are associated with timely initiation of ANC visits in Ethiopia. Similarly, residence, educational status, partner encouragement to go to a clinic for ANC, and timely initiation of ANC are factors significantly associated with adequate $\mathrm{ANC}$ visits.

Although WHO and country-level guidelines recommend initiation of ANC in the first trimester of pregnancy, ${ }^{4}$ only one-fourth of study participants initiated ANC during this time in Ethiopia. This is in line with reports made in 2013 from LMICs, ${ }^{15}$ but is lower than the previous study in Ethiopia (38.8\%). ${ }^{37}$ Similarly, only $33.7 \%$ of study participants initiated ANC during the first trimester of pregnancy in Ethiopia. ${ }^{17}$ The discrepancies of the results might be due to the previous facility-based studies' which used a small sample size, varying definition of timely initiation of ANC visit (cut-of-point $<12$ vs $<16$ weeks), and other sociodemographic factors. Timely initiation of ANC visits provides the opportunity to address pre-existing conditions on the appropriate time, perform a recommended test, and provide or get counselling. ${ }^{48}$ It might also help women perceive the right time for the first ANC booking, ${ }^{37}$ and be informed about its importance. ${ }^{49}$ The low timely initiation of ANC may contribute to the low coverage of continuum of care, high maternal and neonatal morbidity, and high mortality in Ethiopia.

This study reveals that the number of women who had adequate ANC visits in Ethiopia was $43.3 \%$, which is similar to the previous study. ${ }^{50}$ Nevertheless, this result is contrary to results reported by other studies in Ethiopia $\left(33.0 \%{ }^{23}\right.$ and $\left.77.7 \%\right){ }^{17}$ The underutilisation of ANC could be, consequently, the possible reason for high maternal and child mortality in Ethiopia. Low utilisation of services might be attributed to distance; service availability, and accessibility variations among regions, ${ }^{51}$ low awareness, ${ }^{52}$ and quality of services, ${ }^{17}$ and beliefs about pregnancy and other cultural beliefs. ${ }^{53}$ These show that Ethiopia has to improve the coverage of four or more ANC to achieve its target of health sectors transformation plan II.

The results of this study show that the odds of timely initiation of ANC in the first trimester of pregnancy are lower among rural residents compared with urban dwellers. Similarly, rural residents are less likely to initiate ANC visits during the first trimester in Ethiopia, ${ }^{23} 26$ Nigeria ${ }^{30}$ and Myanmar. ${ }^{31}$ Quality of ANC services, travel expenses due to the distance of healthcare facilities (inaccessibility), access to information and knowledge about the benefit of timing, and adequate ANC might be mediating such results. ${ }^{20}$ Furthermore, inadequate availability and unequal distribution of health facilities and healthcare providers might contribute to the variation of timely initiation of ANC visit between rural and urban settings. The result could be a good input for policies to support rural women to improve initiation of ANC in the first trimester of gestation.

Furthermore, the odds of timely initiation of the first ANC visit among Addis Ababa women is twice that of the Tigray region and is lower in SNNP. This finding is in line with other findings in Ethiopia, and Nigeria. ${ }^{23} 2630$ Possible explanations of the results might be attributed to the varying distribution of maternal health services, a scarce resource in underprivileged settings, and concentration in urban and nearby areas such as city administration of the country. This implies further research is needed to thoroughly identify factors contributing to these regional disparities. 
Also, this study indicates that Muslim women initiated ANC late compared with their Orthodox counterparts. This coincides with other findings in Ethiopia and Nigeria which attest that Christians initiate ANC in the first trimester of pregnancy compared with their Muslim counterparts. ${ }^{54}$ The finding highlights tailored intervention for Muslim women plays a crucial role to improve the timeliness of their ANC visits.

As regards the educational status, the results of this study indicate that it plays a key role in the initiation of ANC in the first trimester of pregnancy. The finding is similar to previous studies. ${ }^{9} 2326$ The importance of ANC visits (ie, timing and adequate numbers of visits) might be gained through formal education because educated women could appreciate problems related to late initiation of ANC. Information received about the correct time of ANC booking shows that it is positively associated with the timely initiation of ANC. ${ }^{16}$ The odds of initiating ANC during the first trimester of pregnancy among multipara women is lower compared with primipara women. Similarly, studies indicated that multipara women are less likely to tend to initiate ANC visits in the first trimester of gestation. ${ }^{96}$ Pregnancy experiences and the burden of childcare may contribute to late initiation of ANC. ${ }^{26}$

Besides, the results show that a woman who is encouraged by her partner to go to a clinic for an ANC visit has an increased odds of ANC initiation in the first trimester compared with her counterpart who is not encouraged to go to a clinic for the visit. Similarly, not being supported by a husband or a partner is identified as a factor associated with a later ANC enrolment. ${ }^{56}$ This might be because women in Ethiopia and many other LMICs do not freely decide on matters of their own life. Men's tremendous control over their partners in LMICs includes whether or not their spouses will use available health services. ${ }^{57}$ Therefore, male partner involvement in decision-making about the ANC process should be highlighted during policy and programme designing and implementation.

On the other hand, it has been found out that timely ANC initiation has higher odds of receiving adequate ANC visits and is much more likely to receive WHOrecommended contents of ANC compared with delayed ANC initiation. ${ }^{8} 9$ Similarly, in previous studies, early initiation of ANC visits predicted the use of 4 plus ANC visits. ${ }^{27}$ Thus, improving early arrival in the first trimester for services, and motivating mothers that begin ANC to confirm continuity is crucial ${ }^{23} 58$ because drop-out from ANC is significantly associated with not being informed about pregnancy complications. ${ }^{59}$ Further, women who come to health centres early during pregnancy have enough time for consecutive visits and the information they get may facilitate the continuity of care. Hence, timely initiation of ANC visits positively influences the continuity of services utilisation, provided that women are satisfied and many other factors are controlled.

Nevertheless, women who live in a rural part of Ethiopia have lower odds of adequate ANC visits compared with urban women. This result is consistent with previous studies. ${ }^{1724}$ This might be due to the distance from health facilities. ${ }^{5160}$ The geographical locations of the women, indeed, play important role in the adequacy of ANC visits. For instance, a woman in Afar and SNNP had lower odds of adequate ANC visits compared with those in the Tigray region, but Addis Ababa women had the higher odds of adequate ANC visits. This finding is consistent with a study that revealed $25 \%$ of the variability in having at least four ANC visits was accounted for by region of their living. ${ }^{51}$ Subsequently, variations in odds of the adequate ANC might be due to pre-existing health system; that is, variation of healthcare providers, training of focused ANC components which hinders its implementation, resource and women's counselling. This study highlights the gap in the attainment of the recommended four or more ANC visits regarding women's place of residence.

This study revealed that the educational level of women increased the odds of adequate ANC visits. Similarly, the educational status of women is associated with adequate ANC utilisation. ${ }^{24}{ }^{27}$ Moreover, the education of girls reduces gender disparity, while it improves wealth and autonomy which favour the use of healthcare, and enhances health-seeking, and empowerment to access care. ${ }^{6162}$ The finding highlighted that female education played a crucial role, particularly for those who are rural residents, to improve coverage of four or more ANC visits.

Finally, the odds of adequate ANC visits among women who are encouraged by their partners to go to ANC clinic is found to be as twice as that of their counterparts who are not encouraged by their partners. This means a partner's support or involvement is deemed to facilitate adequate ANC visits. ${ }^{27}$ This might be due to the common fact that women's access to skilled care is influenced by spousal support and decisions of maternal health services. ${ }^{63}$ Such practice plays a considerable role in patriarchal systems in many parts of Africa wherein men have the power to make decisions including about their wives' use of maternal health services. ${ }^{64}$ It can be concluded that spousal encouragement or support is one of the motivational factors for women's healthcare utilisation, in general, and pregnancy-related care (such as ANC), in particular. Women who have family or spousal or partner support are more likely to care for themselves and the pregnancy. The spousal or partner support could be viewed in terms of moral (psychological), financial, accompanying and transportation.

To end, this study showed that between clusters, residual heterogeneity was of greater significance than was the impact of the partner involved in decision making about the ANC process for understanding variations in the odds of timely initiation of ANC visit. Similarly, the residual heterogeneity between clusters was of greater importance than the influence of the partner involved in decision making about the ANC process for understanding variations in the odds of attending four or more ANC visits. The finding highlighted that community-level factors are priority issues during the designing and implementation of policies and programmes. 


\section{Strength and limitations}

The community-based panel study design was implemented to collect nationally representative data and used female and household variables' weighting which makes the findings generalisable to women of reproductive age in Ethiopia. The use of a multilevel logistic regression model considered the hierarchical nature of the data that included women nested within EAs with analysis as well as community and individual-level factors. However, the authors duly acknowledged some limitations of this study. The loss of follow-up (ie, missing vital information due to attrition by being unreachable), together with recall error as well as tendency to give desirable information by women, could result in bias in the findings. Moreover, the Performance Monitoring for Action (PMA) Ethiopia also did not collect information on barriers to utilisation of health services, such as women's perception and awareness about ANC, exposure to media, healthcare delivery, and providers' related factors and the ethnicity of the women.

\section{Conclusions}

Only one-fourth of study participants initiated ANC during the first trimester of pregnancy in Ethiopia which is lower than the target recommended for LMICS. The numbers of women who had four or more ANC visits were low. Timeliness of ANC visit depends on the place of residence, regions of dwelling, religion, level of education, birth events and partner involvement in decision making about the ANC process. Similarly, place of residence, regions of dwelling, level of education, partner involvement in decision making about the ANC process, and timeliness of ANC visits were significantly associated with the adequacy of ANC visits. Concerted efforts on tailored interventions for rural residents, female education and partner involvement are potential remedies for timeliness and adequacy of ANC visit(s).

Acknowledgements We would like to thank PMA Ethiopia for giving us the data.

Contributors The first author (KS) planned, conducted, prepared a report of the work and acts as guaranter. KS wrote the first draft of the manuscript and BM, TG, MD and AS contributed to subsequent revisions. KS, BM, TG, MD and AS conceived, designed, executed and participated in the data acquisition, analysis and interpretation of the findings. KS, BM, TG, MD and AS participated in approving the final version to be published, journal selection to which the article has been submitted and agreed to be responsible for all aspects of the work.

Funding The authors have not declared a specific grant for this research from any funding agency in the public, commercial or not-for-profit sectors.

Competing interests None declared.

Patient and public involvement Patients and/or the public were not involved in the design, or conduct, or reporting, or dissemination plans of this research.

\section{Patient consent for publication Not applicable.}

Ethics approval This study was approved by Ethical approval was obtained from Addis Ababa University, College of Health Sciences' ethical review board (Ref: AAUMF 01-008), and Johns Hopkins University Bloomberg School of Public Health Institutional Review Board (FWA00000287). An informed, voluntary, written and signed consent was obtained from each study participant once they were fully informed. The datasets are anonymized before availing to the public via the www. pmadata.org website as frankly stated in the PMA Ethiopia data use policy. The PMA Ethiopia granted access to datasets after reviewing submitted requests. The privacy, anonymity and confidentiality of data are maintained, that is, any effort to identify individual, household or enumeration areas in the survey and use of data for marketing or commercial ventures is forbidden. Participants gave informed consent to participate in the study before taking part.

Provenance and peer review Not commissioned; externally peer reviewed.

Data availability statement Data are available on reasonable request. Data may be obtained from a third party and are not publicly available. Data used in this analysis are not available for public use currently, but it will be available soon on reasonable request from the PMA Ethiopia website via https://datalab.pmadata.org/ user/login?destination=/download-requests/dataset.

Open access This is an open access article distributed in accordance with the Creative Commons Attribution Non Commercial (CC BY-NC 4.0) license, which permits others to distribute, remix, adapt, build upon this work non-commercially, and license their derivative works on different terms, provided the original work is properly cited, appropriate credit is given, any changes made indicated, and the use is non-commercial. See: http://creativecommons.org/licenses/by-nc/4.0/.

ORCID iD

Kasiye Shiferaw http://orcid.org/0000-0001-6087-1682

\section{REFERENCES}

1 World Health Organization. Health in 2015: from MDGs, millennium development goals to SDGs, sustainable development goals. Geneva, Switzerland: World Health Organization, 2015.

2 World Health Organization. Every newborn action plan: progress report. Geneva, Switzerland: World Health Organization, 2015.

3 Koblinsky M, Moyer CA, Calvert C, et al. Quality maternity care for every woman, everywhere: a call to action. Lancet 2016;388:2307-20.

4 World Health Organization. WHO recommendations on antenatal care for a positive pregnancy experience. World Health Organization, 2016.

5 Gottfredsdottir H, Steingrímsdóttir Póra, Björnsdóttir A, et al. Content of antenatal care: does it prepare women for birth? Midwifery 2016;39:71-7.

6 Vogel JP, Souza JP, Mori R, et al. Maternal complications and perinatal mortality: findings of the world health organization multicountry survey on maternal and newborn health. BJOG 2014;121:76-88.

7 Shattnawi KK, Khader YS, Alyahya MS, et al. Rate, determinants, and causes of stillbirth in Jordan: findings from the Jordan stillbirth and neonatal deaths surveillance (JSANDS) system. BMC Pregnancy Childbirth 2020;20:571.

8 Agha S, Tappis $\mathrm{H}$. The timing of antenatal care initiation and the content of care in Sindh, Pakistan. BMC Pregnancy Childbirth 2016;16:190.

9 Jiwani SS, Amouzou-Aguirre A, Carvajal L, et al. Timing and number of antenatal care contacts in low and middle-income countries: analysis in the countdown to 2030 priority countries. J Glob Health 2020;10:010502.

10 Carroli G, Rooney C, Villar J. How effective is antenatal care in preventing maternal mortality and serious morbidity? An overview of the evidence. Paediatr Perinat Epidemiol 2001;15:1-42.

11 Federal Ministry of Health E. Antenatal care module: 13. providing focused antenatal care. Addis Ababa: Federal ministry of health, Ethiopia, 2008.

12 Tunçalp Ö, Pena-Rosas JP, Lawrie T, et al. WHO recommendations on antenatal care for a positive pregnancy experience-going beyond survival. BJOG 2017;124:860-2.

13 Lincetto S-AO, Gomez P, Munjanja S. Antenatal care. Opportunities for Africa's newborns: practical data, policy and programmatic support for newborn care in Africa. World Health Organisation, 2006.

14 Arsenault C, Jordan K, Lee D, et al. Equity in antenatal care quality: an analysis of 91 national household surveys. Lancet Glob Health 2018;6:e1186-95.

15 Moller A-B, Petzold M, Chou D, et al. Early antenatal care visit: a systematic analysis of regional and global levels and trends of coverage from 1990 to 2013. Lancet Glob Health 2017;5:e977-83.

16 Gidey G, Hailu B, Nigus K, et al. Timing of first focused antenatal care booking and associated factors among pregnant mothers who attend antenatal care in central zone, Tigray, Ethiopia. BMC Res Notes 2017;10:608.

17 Yaya S, Bishwajit G, Ekholuenetale M, et al. Timing and adequate attendance of antenatal care visits among women in Ethiopia. PLOS One 2017;12:e0184934. 
18 Jo Y, Alland $\mathrm{K}$, Ali $\mathrm{H}$, et al. Antenatal care in rural Bangladesh: current state of costs, content and recommendations for effective service delivery. BMC Health Serv Res 2019;19:861.

19 Tesfaye G, Loxton D, Chojenta C, et al. Delayed initiation of antenatal care and associated factors in Ethiopia: a systematic review and meta-analysis. Reprod Health 2017;14:150.

20 Wolde HF, Tsegaye AT, Sisay MM. Late initiation of antenatal care and associated factors among pregnant women in Addis Zemen primary hospital, South Gondar, Ethiopia. Reprod Health 2019;16:73.

21 Tikmani SS, Ali SA, Saleem S, et al. Trends of antenatal care during pregnancy in low- and middle-income countries: findings from the global network maternal and newborn health registry. Semin Perinatol 2019;43:297-307.

22 Kanyangarara M, Munos MK, Walker N. Quality of antenatal care service provision in health facilities across sub-Saharan Africa: evidence from nationally representative health facility assessments. J Glob Health 2017;7:021101.

23 Muchie KF. Quality of antenatal care services and completion of four or more antenatal care visits in Ethiopia: a finding based on a demographic and health survey. BMC Pregnancy Childbirth 2017;17:1-7.

24 Mekonnen T, Dune T, Perz J, et al. Trends and determinants of antenatal care service use in Ethiopia between 2000 and 2016. Int J Environ Res Public Health 2019;16:748.

25 Yeoh PL, Hornetz K, Dahlui M. Antenatal care utilisation and content between low-risk and high-risk pregnant women. PLoS One 2016;11:e0152167.

26 Teshale AB, Tesema GA. Prevalence and associated factors of delayed first antenatal care booking among reproductive age women in Ethiopia; a multilevel analysis of EDHS 2016 data. PLoS One 2020;15:e0235538

27 Tizazu MA, Asefa EY, Muluneh MA, et al. Utilizing a minimum of four antenatal care visits and associated factors in Debre Berhan town, North Shewa, Amhara, Ethiopia, 2020. Risk Manag Healthc Policy 2020;13:2783-91.

28 Grum T, Brhane E. Magnitude and factors associated with late antenatal care booking on first visit among pregnant women in public health centers in central zone of Tigray Region, Ethiopia: a cross sectional study. PLoS One 2018;13:e0207922.

29 Njiku F, Wella H, Sariah A, et al. Prevalence and factors associated with late antenatal care visit among pregnant women in Lushoto, Tanzania. Tanzan J Health Res 2017;19.

30 Aliyu AA, Dahiru T. Predictors of delayed Antenatal Care (ANC) visits in Nigeria: secondary analysis of 2013 Nigeria Demographic and Health Survey (NDHS). Pan Afr Med J 2017;26.

31 Aung TZ, WM O, Khaing W. Late initiation of antenatal care and its determinants: a hospital based cross-sectional study. Int J Comm Med Public Health 2017;3:900-5.

32 Manzi A, Munyaneza F, Mujawase F, et al. Assessing predictors of delayed antenatal care visits in Rwanda: a secondary analysis of Rwanda demographic and health survey 2010. BMC Pregnancy Childbirth 2014;14:1-8.

33 Nimi T, Fraga S, Costa D, et al. Prenatal care and pregnancy outcomes: a cross-sectional study in Luanda, Angola. Int J Gynaecol Obstet 2016:135:S72-8.

34 Adewuyi EO, Auta A, Khanal V, et al. Prevalence and factors associated with underutilization of antenatal care services in Nigeria: a comparative study of rural and urban residences based on the 2013 Nigeria demographic and health survey. PLoS One 2018:13:e0197324.

35 Singh K, Story WT, Moran AC. Assessing the continuum of care pathway for maternal health in South Asia and Sub-Saharan Africa. Matern Child Health J 2016;20:281-9.

36 Mchenga M, Burger R, von Fintel D. Examining the impact of WHO's focused antenatal care policy on early access, underutilisation and quality of antenatal care services in Malawi: a retrospective study. BMC Health Serv Res 2019;19:295.

37 Tola W, Negash E, Sileshi T, et al. Late initiation of antenatal care and associated factors among pregnant women attending antenatal clinic of llu Ababor Zone, Southwest Ethiopia: a cross-sectional study. PLoS One 2021;16:e0246230.

38 Zimmerman L, Desta S, Yihdego M, et al. Protocol for PMA-Ethiopia: a new data source for cross-sectional and longitudinal data of reproductive, maternal, and newborn health. Gates Open Res 2020;4:126.

39 Central Statistical Agency(CSA)[Ethiopia] and ICF. Ethiopia demographic and health survey 2016: key indicators report. Addis Ababa, Ethiopia, and Rockville, Maryland, USA: CSA and ICF, 2016.
40 Golini A, Said M, Casacchia O, et al. Migration and urbanization in Ethiopia, with special reference to Addis Ababa. Addis Ababa: CSA, 2001.

41 Ekholuenetale M, Benebo FO, Idebolo AF. Individual-, household-, and community-level factors associated with eight or more antenatal care contacts in Nigeria: evidence from demographic and health survey. PLoS One 2020;15:e0239855.

42 Mekonnen ZA, Lerebo WT, Gebrehiwot TG, et al. Multilevel analysis of individual and community level factors associated with institutional delivery in Ethiopia. BMC Res Notes 2015;8:376.

43 Siegmunt O. Intraclass correlation, median odds ratio, and ecological reliability. Neighborhood disorganization and social control. Springer, 2016: 43-5.

44 Merlo J, Chaix B, Ohlsson $\mathrm{H}$, et al. A brief conceptual tutorial of multilevel analysis in social epidemiology: using measures of clustering in multilevel logistic regression to investigate contextual phenomena. J Epidemiol Community Health 2006;60:290-7.

45 Austin PC, Stryhn H, Leckie G, et al. Measures of clustering and heterogeneity in multilevel poisson regression analyses of rates/ count data. Stat Med 2018;37:572-89.

46 Halonen JI, Kivimäki M, Pentti J, et al. Quantifying neighbourhood socioeconomic effects in clustering of behaviour-related risk factors: a multilevel analysis. PLoS One 2012;7:e32937.

47 Shiferaw K, Mengistie B, Gobena T, et al. Extent of received antenata care components in Ethiopia: a community-based panel study. Int $J$ Womens Health 2021;13:803-13.

48 EBCOG Scientific Committee. The public health importance of antenatal care. Facts Views Vis Obgyn 2015;7:5

49 Kisuule I, Kaye DK, Najjuka F, et al. Timing and reasons for coming late for the first antenatal care visit by pregnant women at Mulago hospital, Kampala Uganda. BMC Pregnancy Childbirth 2013;13:121.

50 Basha GW. Factors affecting the utilization of a minimum of four antenatal care services in Ethiopia. Obstet Gynecol Int 2019;2019:1-6.

51 Tegegne TK, Chojenta C, Getachew T, et al. Antenatal care use in Ethiopia: a spatial and multilevel analysis. BMC Pregnancy Childbirth 2019;19:399.

52 Aziz Ali S, Aziz Ali S, Feroz A, et al. Factors affecting the utilization of antenatal care among married women of reproductive age in the rural Thatta, Pakistan: findings from a community-based case-control study. BMC Pregnancy Childbirth 2020;20:355.

53 Simkhada B, Teijlingen ERvan, Porter M, et al. Factors affecting the utilization of antenatal care in developing countries: systematic review of the literature. $J$ Adv Nurs 2008;61:244-60.

54 Seid A, Ahmed M. Survival time to first antenatal care visit and its predictors among women in Ethiopia. PLoS One 2021;16:e0251322.

55 Fagbamigbe AF, Olaseinde O, Fagbamigbe OS. Timing of first antenatal care contact, its associated factors and state-level analysis in Nigeria: a cross-sectional assessment of compliance with the WHO guidelines. BMJ Open 2021:11:e047835

56 Gross K, Alba S, Glass TR, et al. Timing of antenatal care for adolescent and adult pregnant women in south-eastern Tanzania. BMC Pregnancy Childbirth 2012;12:16.

57 Iliyasu Z, Abubakar IS, Galadanci HS, et al. Birth preparedness, complication readiness and fathers' participation in maternity care in a northern Nigerian community. Afr J Reprod Health 2010;14:21-32.

58 Agha S, Tappis H, Sohail Agha HT. The timing of antenatal care initiation and the content of care in Sindh, Pakistan. BMC Pregnancy Childbirth 2016;16:190.

59 Muluneh AG, Kassa GM, Alemayehu GA, et al. High dropout rate from maternity continuum of care after antenatal care booking and its associated factors among reproductive age women in Ethiopia, Evidence from Demographic and Health Survey 2016. PLoS One 2020;15:e0234741.

60 Dadras O, Dadras F, Taghizade Z, et al. Barriers and associated factors for adequate antenatal care among Afghan women in Iran; findings from a community-based survey. BMC Pregnancy Childbirth 2020;20:1-11.

61 Tembon MM, Fort L. Girl's education in the 21st century: Gender equality, empowerment and growth. The World Bank, 2008.

62 Furuta M, Salway S. Women's position within the household as a determinant of maternal health care use in Nepal. Int Fam Plan Perspect 2006;32:017-27.

63 White D, Dynes M, Rubardt M, et al. The influence of intrafamilial power on maternal health care in Mali: perspectives of women, men and mothers-in-law. Int Perspect Sex Reprod Health 2013;39:058-68.

64 Nyandieka LN, Njeru MK, Ng'ang'a Z, et al. Male involvement in maternal health planning key to utilization of skilled birth services in Malindi Subcounty, Kenya. Adv Public Health 2016;2016:1-8. 